\title{
Comparison between Patients Hospitalized with Influenza and COVID-19 at a Tertiary Care Center
}

\author{
Michael W. Donnino, MD ${ }^{1,2}$ (D), Ari Moskowitz, MD ${ }^{1,3}$, Garrett S. Thompson, $\mathrm{MPH}^{7}$, \\ Stanley J. Heydrick, $P h D^{7}$, Rahul D Pawar, $M D^{7}$, Katherine M. Berg, MD', \\ Shivani Mehta, $B A^{\prime}$, Parth V. Patel, BSN, RN ${ }^{7}$, and Anne V. Grossestreuer, PhD ${ }^{7}$
}

${ }^{1}$ Center for Resuscitation Science, Department of Emergency Medicine, Beth Israel Deaconess Medical Center, Boston, MA, USA; ${ }^{2}$ Department of Medicine, Division of Pulmonary Critical Care, Beth Israel Deaconess Medical Center, Boston, MA, USA; ${ }^{3}$ Department of Internal Medicine, Division of Pulmonary, Critical Care, and Sleep Medicine, Beth Israel Deaconess Medical Center, Boston, MA, USA.

BACKGROUND: Widespread reports suggest the characteristics and disease course of coronavirus disease 2019 (COVID-19) and influenza differ, yet detailed comparisons of their clinical manifestations are lacking.

OBJECTIVE: Comparison of the epidemiology and clinical characteristics of COVID-19 patients during the pandemic with those of influenza patients in previous influenza seasons at the same hospital

DESIGN: Admission rates, clinical measurements, and clinical outcomes from confirmed COVID-19 cases between March 1 and April 30, 2020, were compared with those from confirmed influenza cases in the previous five influenza seasons ( 8 months each) beginning September $1,2014$.

SETTING: Large tertiary care teaching hospital in Boston, MA

PARTICIPANTS: Laboratory-confirmed COVID-19 and influenza inpatients

MEASUREMENTS: Patient demographics and medical history, mortality, incidence and duration of mechanical ventilation, incidences of vasopressor support and renal replacement therapy, and hospital and intensive care admissions.

RESULTS: Data was abstracted from medical records of 1052 influenza patients and 582 COVID-19 patients. An average of 210 hospital admissions for influenza occurred per 8-month season compared to 582 COVID-19 admissions over 2 months. The median weekly number of COVID-19 patients requiring mechanical ventilation was 17 (IQR: 4, 34) compared to a weekly median of 1 (IQR: 0, 2) influenza patient ( $p=0.001)$. COVID-19 patients were significantly more likely to require mechanical ventilation (31\% vs $8 \%$ ) and had significantly higher mortality $(20 \%$ vs. $3 \%$; $p<0.001$ for all). Relatively more COVID-19 patients on mechanical ventilation lacked pre-existing conditions compared with mechanically ventilated influenza patients $(25 \%$ vs $4 \%, p<0.001)$. Pneumonia/ARDS secondary to the virus was the predominant cause of mechanical ventilation in COVID-19 patients (94\%) as opposed to influenza (56\%).

Supplementary Information The online version contains supplementary material available at https://doi.org/10.1007/s11606-021-06647-2.

Received August 5, 2020

Accepted January 28, 2021

Published online March 18, 2021
LIMITATION: This is a single-center study which could limit generalization.

CONCLUSION: COVID-19 resulted in more weekly hospitalizations, higher morbidity, and higher mortality than influenza at the same hospital.

KEY WORDS: influenza; COVID-19; mechanical ventilation.

J Gen Intern Med 36(6):1689-95

DOI: $10.1007 / \mathrm{s} 11606-021-06647-2$

(C) Society of General Internal Medicine 2021

\section{INTRODUCTION}

Coronavirus disease 2019 (COVID-19) and influenza are contagious viral diseases characterized by pneumonia and acute respiratory failure in severe cases. Both place a burden on the health care system, but objective granular assessments of their comparative impact on individuals and the healthcare system are lacking in the literature.

The epidemiology, symptomology, and annual public health burden presented by influenza are generally well characterized; however, this is largely through estimates as opposed to comprehensive reporting. ${ }^{1}$ The Centers for Disease Control and Prevention (CDC) estimates that over the past 10 years, between 9 and 45 million people have contracted influenza annually in the USA, with annual hospitalizations ranging from 140,000 to 810,000 and annual mortality ranging from 12,000 to 61,000 (between 0.10 and $0.17 \%$ of all cases), depending on the severity of the season. ${ }^{2}$ Influenza generally achieves community spread nationwide and can strike all age groups, but mortality is consistently higher in patients who are elderly, immunocompromised, and/or have pre-existing comorbidities such as chronic obstructive pulmonary disease, chronic kidney injury, cirrhosis, or cardiac disease. ${ }^{3-9}$ Decades of experience with influenza have led to vaccination programs which can mitigate the impact of influenza in years when the predominant circulating strain is accurately predicted. $^{10}$

Official CDC counts indicate that over one million people in the USA have contracted COVID-19 between the virus's first detected cases through April 30, 2020, with over 150,000 
hospitalizations and over 63,000 deaths reported as of that date. ${ }^{11}$ However, the methods for reporting COVID-19 and influenza cases and outcomes differ in that COVID-19 is directly reported from each state whereas influenza cases are estimates based on reporting from selected hospitals. ${ }^{1}$ Moreover, granular comparisons between these two diseases such as rates and duration of respiratory failure (i.e., mechanical ventilation), need for hospitalization, outcomes of those patients without comorbid disease, frequency of non-pulmonary organ injury, and mortality are lacking, yet essential for understanding these two disease processes.

In this study, we compared the epidemiology and clinical characteristics of COVID-19 patients admitted to a large tertiary care teaching hospital in March and April of 2020 with those of influenza patients admitted to the same hospital over the prior 5 years.

\section{METHODS}

Design. This was a single-center, retrospective study at an urban tertiary care center. We compared patients admitted to the hospital with a positive laboratory test for influenza during five influenza seasons (September-April 2014-2019) to patients with a positive laboratory test for COVID-19 in March and April 2020. This study was reviewed by the Institutional Review Board at Beth Israel Deaconess Medical Center, which determined the study met exempt status. Written informed consent was therefore not required.

Patient Population. Adult (aged $>17$ years) patients were included if they were admitted to the hospital for confirmed influenza or COVID-19 during the time frames of interest. Patients with influenza or COVID-19 were screened for eligibility by International Classification of Diseases, 10th revision (ICD-10) codes (see Appendix) and/or laboratory-based testing. Of the screened patients, only patients with confirmed positivity by laboratory test during that hospitalization were included. Laboratory diagnosis of influenza and SARS-CoV-2 were carried out through specific real-time reverse transcriptase-polymerase chain reaction (RT-PCR) assays of nasal swab specimens. The ICD-10 codes are displayed in Supplement Table S1.

Study Data Acquisition. Patient data included demographics, personal medical histories (e.g., chronic comorbid conditions), laboratory findings on admission, intensive care unit admission status, intensive care unit length of stay, invasive mechanical ventilation, hospital length of stay, and mortality. Key variables including the presence and duration of invasive mechanical ventilation, comorbidities, and mortality were manually reviewed to verify the data. Specifically, trained research assistants reviewed all individual patient charts to gather comorbidity data. A physician, author RP, reviewed classified reasons for intubation by chart review (see supplemental table S2). If the reason for intubation was not clear, author MD adjudicated the reason for intubation. To compute the volume of admissions between the two diseases, weekly counts of hospital admissions and mechanically ventilated patients were assessed during the peak 2 months of influenza seasons from 2014 to 2019 and from March to April 2020 for COVID-19. All cases for which laboratory testing occurred greater than five days after admission were reviewed to determine if the disease was believed to be nosocomial. For other variables (e.g., laboratory values), a sample of randomly chosen data was verified using manual chart review. Definitions for elements of the data abstraction are displayed in Table S2 in the supplementary materials.

Outcomes. We collected a number of continuous and categorical outcomes. The continuous outcomes included the duration of mechanical ventilation, the duration of the patient's index hospital stay for that year, the number of weekly admissions, and the number of weekly incident mechanical ventilation. The categorical outcomes included the incidence of mechanical ventilation, shock, renal replacement therapy, hospital readmissions, ICU admissions, and in-hospital mortality, as well as the reason for mechanical ventilation the proportion of patients on mechanical ventilation with no major comorbidities, and the proportion of overall patients with no major comorbidities.

Data Analysis. Continuous data are presented as medians with interquartile ranges and categorical data are presented as counts and percentages. For continuous outcomes, medians were compared using a Wilcoxon rank-sum test. For categorical outcomes, proportions were compared using chi-squared or Fisher's exact tests, as appropriate. A subgroup analysis was performed on patients intubated for COVID-19 and influenza pneumonia/ARDS. All analyses were done using Stata 14.2 (College Station, TX) and a $p$ value $<0.05$ was considered statistically significant.

\section{RESULTS}

\section{Overview of COVID-19 and Influenza Admis- sions with Key Outcomes}

In total, 1855 patients were identified and 1634 were included in the study (Fig. 1). Of those included in the study, 582 patients had laboratory-confirmed SARS-CoV-2 and 1052 patients had laboratory-confirmed influenza. An overview of admissions, intensive care unit admissions, receipt of mechanical ventilation, and mortality is illustrated per influenza season compared to COVID-19 (Table 1). The total admissions for influenza per eight-month influenza season averaged 210 (95\% CI: 150-271) compared to a total of 582 COVID-19 


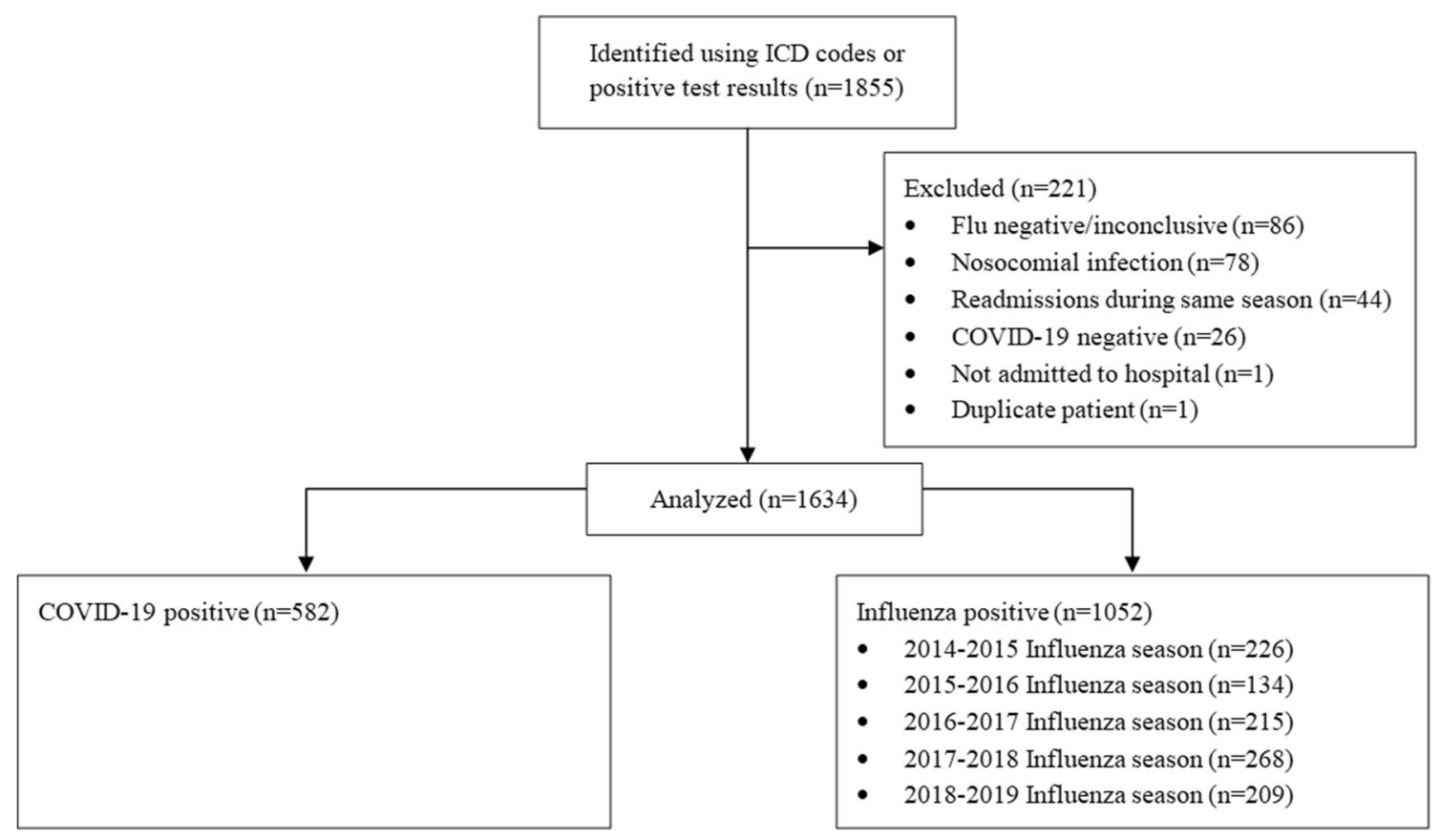

Figure 1 Patient inclusion/exclusion criteria.

admissions over the 2-month COVID-19 study period. A total of 174 COVID-19 patients who were admitted in March and April 2020 were placed on mechanical ventilation compared to 84 patients admitted over five seasons of influenza. The proportion of admitted patients who received mechanical ventilation was significantly higher for COVID-19 patients compared to that for influenza patients (30\% [174/582] versus $8 \%$ [84/1052], $p<0.001)$. One hundred and nineteen patients (20\%) admitted in March and April 2020 with COVID-19 died compared to 34 patients (3\%) over five seasons of influenza $(p<0.001)$. Figure 2 illustrates the rates of COVID-19 admissions compared to influenza admissions per season in

Table 1 Number of Total Patients, Deaths, ICU Admissions, and Patients Requiring Mechanical Ventilation in Five Influenza Seasons (September 2014-May 2019) and the Initial 2 Months (March and April 2020) of COVID-19

\begin{tabular}{|c|c|c|c|c|}
\hline Season & $\begin{array}{l}\text { Total } \\
\text { patients, } \\
n\end{array}$ & $\begin{array}{l}\text { Mortality, } \\
n(\%)\end{array}$ & $\begin{array}{l}\text { ICU } \\
\text { admission, } \\
n(\%)\end{array}$ & $\begin{array}{l}\text { Mechanical } \\
\text { ventilation, } \\
n(\%)\end{array}$ \\
\hline \multicolumn{5}{|c|}{ Influenza (season-September to April: 8 months) } \\
\hline $\begin{array}{l}2014 \\
2015\end{array}$ & 226 & $5(2)$ & $54(24)$ & $18(8)$ \\
\hline $\begin{array}{l}2015- \\
2016\end{array}$ & 134 & $3(2)$ & $37(28)$ & $15(11)$ \\
\hline $\begin{array}{l}2016- \\
2017\end{array}$ & 215 & 7 (3) & $34(16)$ & $9(4)$ \\
\hline $\begin{array}{l}2017- \\
2018\end{array}$ & 268 & $10(4)$ & $60(22)$ & $25(9)$ \\
\hline $\begin{array}{l}2018- \\
2019\end{array}$ & 209 & $9(4)$ & $46(22)$ & $17(8)$ \\
\hline Total & 1052 & $34(3)$ & $231(22)$ & $84(8)$ \\
\hline \multicolumn{5}{|c|}{ COVID-19 (season-March to April: 2 months) } \\
\hline 2020 & 582 & $119(20)$ & $251(43)$ & $174(30)$ \\
\hline
\end{tabular}

$p<0.001$ for comparison between total influenza mortality and COVID19 mortality, comparison between total influenza ICU admissions and COVID-19 admissions, and comparison between total influenza mechanical ventilation and COVID-19 ventilation the busiest 2 months of each influenza season. The timing of local social distancing measures for COVID-19 is also indicated in Figure 2. There was a median of 70 (IQR: 13, 1434) COVID-19 patients admitted per week compared to 16 (IQR: 8,20 ) during the busiest 2 months of the influenza season $(p=0.044)$. In addition, the number of COVID-19 patients placed on mechanical ventilation per week was 17 (IQR: 4, $34)$ compared to a median of 1 (IQR: 0,2 ) influenza patient $(p<0.001)$.

\section{Demographics and Clinical Characteristics}

Patient demographics and medical history are summarized in Table 2. Laboratory values for all patients and patients who required mechanical ventilation are displayed in supplement Tables S3 and S4. There were 304 (52\%) males admitted with COVID-19 and 481 (46\%) males admitted with influenza. The median age for COVID-19 admissions was 66 (IQR: 52, 77) and 68 (IQR: 56, 80) for influenza admissions. The median body mass index for COVID-19 patients was 29.3 (IQR: 25.7, 34.1) compared to 27.3 (IQR: 23.6, 32.3) for influenza. Past medical history is noted between the two groups in Table 2 for the COVID-19 cohort and the 5 seasons of influenza. There were significantly more patients with no major comorbidities in the COVID-19 group than in the influenza patients (113 [19\%] vs 90 [9\%], $p<0.001)$.

In patients who received mechanical ventilation, $55 \%$ of the COVID-19 patients were male, compared to $57 \%$ of the influenza patients. The median age for COVID-19 patients on mechanical ventilation was 66 (IQR: 54, 74), compared with 68 (IQR: 57,81 ) for influenza patients on mechanical ventilation. The median body mass index for COVID-19 patients on mechanical ventilation was 30.8 (IQR: 27.3, 


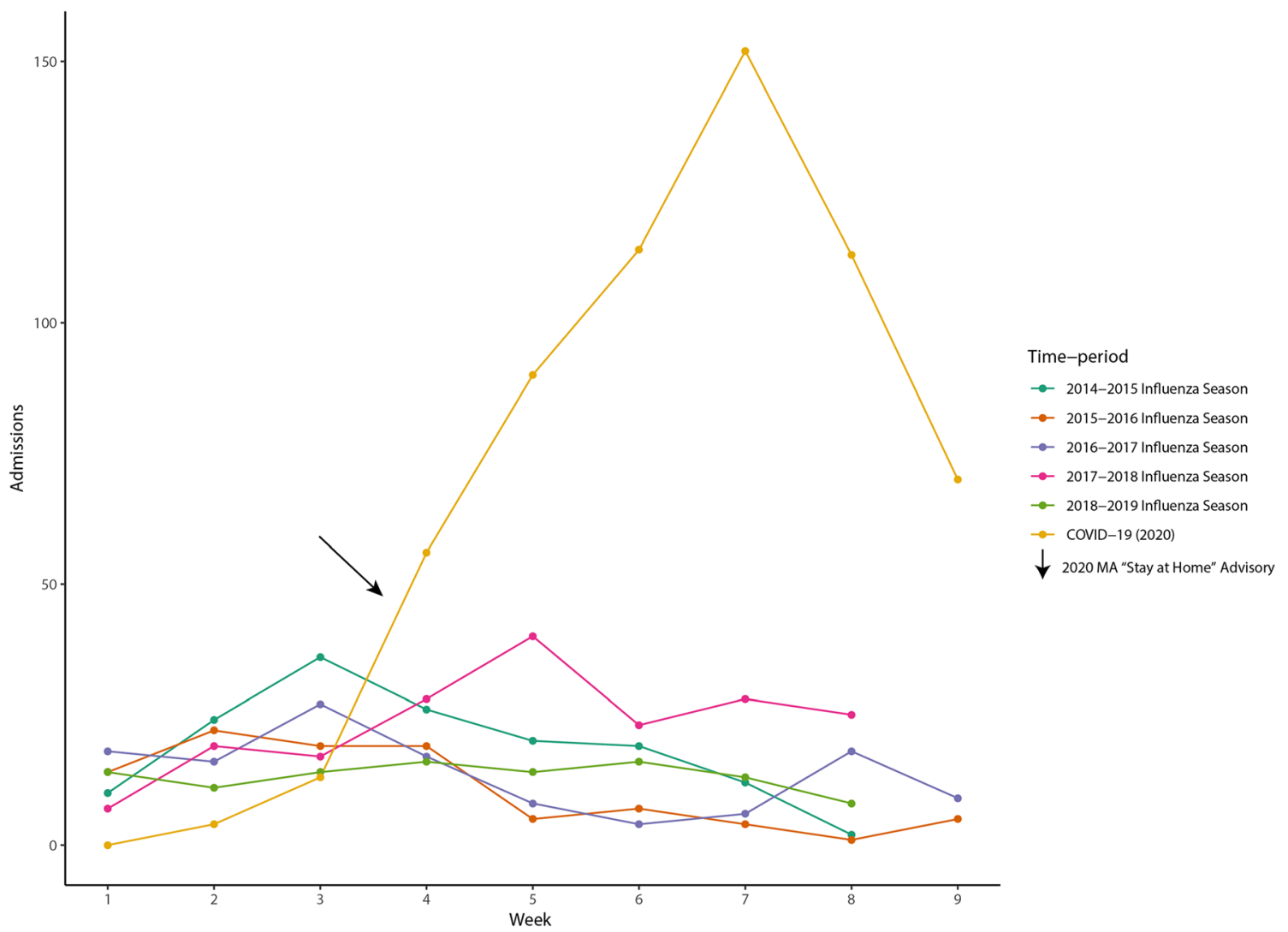

Figure 2 Comparison of COVID-19 hospital admissions during the 9 weeks in March-April 2020 and influenza hospital admissions for the peak 9 weeks in five different seasons. The arrow denotes the approximate timing of the "stay at home" order proclaimed by the Governor of Massachusetts on March 24, 2020.

36.1) compared to 27.6 (IQR: 23.3, 32.6) for influenza patients. COVID-19 patients who were mechanically ventilated were statistically significantly more likely to not have major comorbidities compared to influenza patients on mechanical ventilation (44 [25\%] vs $3[4 \%], p<0.001)$. The reasons for intubation and subsequent mechanical ventilation are displayed in Table 3. Overall, the reasons for intubation in COVID-19 patients were different than those intubated for

Table 2 Demographics and Past Medical History

\begin{tabular}{|c|c|c|c|c|c|c|}
\hline & \multicolumn{3}{|l|}{ All patients } & \multicolumn{3}{|c|}{ Mechanically ventilated patients } \\
\hline & $\begin{array}{l}\text { COVID-19 } \\
(n=583)\end{array}$ & $\begin{array}{l}\text { Seasonal influenza } \\
(n=1052)\end{array}$ & $\begin{array}{l}p \\
\text { value }\end{array}$ & $\begin{array}{l}\text { COVID-19 } \\
(n=174)\end{array}$ & $\begin{array}{l}\text { Seasonal influenza } \\
(n=84)\end{array}$ & $\begin{array}{l}p \\
\text { value }\end{array}$ \\
\hline \multicolumn{7}{|l|}{ Demographics } \\
\hline Male: $n(\%)$ & $304(52)$ & $481(46)$ & 0.013 & $96(55)$ & $48(57)$ & 0.765 \\
\hline Age: median years (IQR) & $66(52,77)$ & $68(56,80)$ & 0.005 & $66(54,74)$ & $68(57,81)$ & 0.086 \\
\hline Body mass index: median (IQR)* & $\begin{array}{l}29.3(25.7, \\
34.0)\end{array}$ & $27.3(23.6,32.3)$ & $<0.001$ & $\begin{array}{l}30.4(27.3 \\
35.5)\end{array}$ & $28.0(23.3,32.9)$ & $<\mathbf{0 . 0 0 1}$ \\
\hline \multicolumn{7}{|l|}{ Past medical history } \\
\hline No major comorbidities: $n(\%)$ & $113(19)$ & $90(9)$ & $<0.001$ & $44(25)$ & $3(4)$ & $<0.001$ \\
\hline Cardiovascular disease: $n(\%)$ & $141(24)$ & $373(35)$ & $<0.001$ & $37(21)$ & $27(32)$ & 0.058 \\
\hline $\begin{array}{l}\text { Respiratory disease (COPD, asthma): } n \\
(\%)\end{array}$ & $119(20)$ & $299(28)$ & $<0.001$ & $29(17)$ & $30(36)$ & $<0.001$ \\
\hline Dementia: $n(\%)$ & $69(12)$ & $84(8)$ & 0.010 & $10(6)$ & $5(6)$ & $>0.999$ \\
\hline Stroke: $\mathrm{n}(\%)$ & $50(9)$ & $90(9)$ & 0.988 & $8(5)$ & $1(1)$ & 0.279 \\
\hline $\begin{array}{l}\text { Immunosuppressed (HIV/ AIDS, } \\
\text { transplant): } n(\%)\end{array}$ & $10(2)$ & $96(9)$ & $<0.001$ & $3(2)$ & $4(5)$ & 0.220 \\
\hline Hypertension: $n(\%)$ & $352(60)$ & $638(61)$ & 0.915 & $106(61)$ & $48(57)$ & 0.562 \\
\hline Diabetes mellitus: n (\%) & $210(36)$ & $298(28)$ & 0.001 & $69(39)$ & $30(36)$ & 0.542 \\
\hline Renal disease: $n(\%)$ & $110(19)$ & $211(20)$ & 0.562 & $30(17)$ & $15(18)$ & 0.903 \\
\hline Liver disease: $n(\%)$ & $25(4)$ & $73(7)$ & 0.031 & $11(6)$ & $1(1)$ & 0.111 \\
\hline Cancer: $n(\%)$ & $95(16)$ & $278(27)$ & $<0.001$ & $32(18)$ & $24(29)$ & 0.063 \\
\hline Morbid obesity (BMI>40) & $52(11)$ & $77(8)$ & 0.062 & $23(14)$ & $4(6)$ & 0.113 \\
\hline Alcohol use disorder: $n(\%)$ & $33(6)$ & $147(14)$ & $<0.001$ & $16(9)$ & $7(8)$ & $>0.999$ \\
\hline Tobacco: $n(\%)$ & $107(18)$ & $211(20)$ & 0.405 & $29(17)$ & $22(26)$ & 0.072 \\
\hline
\end{tabular}

*Body Mass Index were missing from 77 patients with COVID-19 and 22 patients with influenza 
Table 3 Reasons for Respiratory Failure in Patients with COVID19 in March-April 2020 Compared to Patients with Influenza in Five Influenza Seasons (September 2014-May 2019)

\begin{tabular}{llll}
\hline \hline & $\begin{array}{l}\text { COVID-19 } \\
(\boldsymbol{n}=\mathbf{1 7 5})\end{array}$ & $\begin{array}{l}\text { Influenza } \\
(\boldsymbol{n}=\mathbf{8 4})\end{array}$ & $\begin{array}{l}\boldsymbol{p} \\
\text { value }\end{array}$ \\
\hline $\begin{array}{l}\text { Pneumonia/acute respiratory } \\
\text { distress syndrome: } n \text { (\%) }\end{array}$ & $164(94)$ & $47(56)$ & $<0.001$ \\
$\begin{array}{l}\text { Exacerbation of pre-existing } \\
\text { health condition }\end{array}$ & $0(0)$ & $12(14)$ & \\
(e.g., asthma, chronic lung & & & \\
disease, etc.): $n$ (\%) & & $17(30)$ \\
Other causes: $n$ (\%) & $10(6)$ & $4(5)$ & \\
Neurological (e.g., & $1(1)$ & $8(10)$ & \\
intracranial bleed) & $2(1)$ & $2(1)$ & \\
Surgery/procedure & $2(1)$ & $1(1)$ & \\
Cardiac arrest & $1(1)$ & $1(1)$ & \\
$\begin{array}{l}\text { Gastrointestinal hemorrhage } \\
\text { Hemodynamic instability }\end{array}$ & $0(0)$ & $0(0)$ & \\
Trauma & $1(1)$ & \\
Unknown & $3(2)$ & \\
$\begin{array}{l}\text { Multifactorial causes not } \\
\text { deemed to be primarily }\end{array}$ & & \\
pneumonia & & \\
\hline
\end{tabular}

influenza $(p<0.001)$. Pneumonia and/or acute respiratory distress syndrome (ARDS) was the reason for $94 \%$ of intubations in COVID-19 patients, while this accounted for only $56 \%$ of intubations in influenza patients. Additionally, no COVID-19 patients received mechanical ventilation due to exacerbation of pre-existing conditions compared to $14 \%$ of influenza cases who received mechanical ventilation for this indication.

\section{Additional Outcomes}

Table 4 summarizes additional clinical outcomes including duration of index hospital admission, duration of mechanical ventilation, provision of vasopressor support, and provision of

Table 4 Clinical Outcomes in COVID-19 Patients Treated between March and April 2020 and Influenza Patients Treated During five Influenza Seasons (September 2014-May 2019)

\begin{tabular}{llll}
\hline \hline Outcome & $\begin{array}{l}\text { COVID-19 } \\
\text { Spring, } \\
\mathbf{2 0 2 0}(\boldsymbol{n}=\end{array}$ & $\begin{array}{l}\text { Influenza } \\
\mathbf{2 0 1 4 - 2 0 1 9} \\
(\boldsymbol{n}=\mathbf{1 0 5 2})\end{array}$ & $\begin{array}{l}\boldsymbol{p} \\
\text { value }\end{array}$ \\
& $8(4,16)$ & $4(2,7)$ & $<0.001$ \\
\hline $\begin{array}{l}\text { Index hospital length of } \\
\text { stay:median days (IQR) }\end{array}$ & $174(30)$ & $84(8)$ & $<0.001$ \\
$\begin{array}{l}\text { Mechanical ventilation: } n \\
\text { (\%) }\end{array}$ & $14.3(7.9$, & $.3(1.7,7.9)$ & $<0.001$ \\
$\begin{array}{l}\text { Time on mechanical } \\
\text { ventilation-intubated pa- } \\
\text { tients:* median days (IQR) }\end{array}$ & $22.5)$ & & \\
$\begin{array}{l}\text { Time on mechanical } \\
\text { ventilation-intubated pa- }\end{array}$ & $14.7(8.5$, & $3.8(2.0$, & $<0.001$ \\
$\begin{array}{l}\text { tients with pneumonia/ } \\
\text { ARDS:* median days (IQR) }\end{array}$ & $22.7)$ & $12.5)$ & \\
$\begin{array}{l}\text { Shock - on vasopressors } \\
\text { during hospitalization**: } n \\
\text { (\%) }\end{array}$ & $177(30)$ & $74(7)$ & $<0.001$ \\
$\begin{array}{l}\text { Renal replacement therapy: } \\
n(\%)\end{array}$ & $50(9)$ & $12(1)$ & $<0.001$ \\
$\begin{array}{l}\text { Readmitted within } 30 \\
\text { days: } n \text { (\%) }\end{array}$ & $29(5)$ & $15(1)$ & $<0.001$ \\
\begin{tabular}{l} 
Hospital mortality: $n(\%)$ \\
\hline
\end{tabular} & $119(20)$ & $34(3)$ & $<0.001$ \\
\hline
\end{tabular}

*Including reintubation time

**Measured at the encounter level ( $n=613$ for COVID-19; $n=1070$ for influenza) renal replacement therapy. Patients with COVID-19 had a statistically significantly longer median duration of mechanical ventilation than patients with influenza (14.3 [IQR: 7.9, 22.5] vs 3.3 [IQR: 1.7, 7.9] days, $p<0.001)$. Fifty COVID-19 patients (9\%) received renal replacement therapy during their hospitalization, compared to $12(1 \%)$ of influenza patients $(p<0.001)$. One hundred and seventy-seven COVID-19 patients $(30 \%)$ received vasopressor support during their hospitalization, compared to 74 (7\%) of influenza patients $(p<0.001)$. Six of the COVID-19 deaths during the 2-month COVID-19 window were patients who had no major comorbidities (1\% of total patients), whereas potentially only three deaths during 5 years of influenza seasons were patients without comorbidities $(0.3 \%$ of total patients). No deaths among those without major comorbidities were reported in the most recent influenza season. Of note, one of the three deaths in the influenza cohort occurred in a patient who presented with and died from widespread arterial and venous thrombus of unclear etiology and had not seen a physician in many years thus making a determination of underlying conditions difficult but was given a default labeling of healthy.

In the subgroup analysis of patients with COVID-19 and influenza pneumonia, we found that those with COVID-19 were younger (66 years vs 70 years, $p 0.024$ ), had less preexisting comorbid disease (26\% vs $9 \%, p$ value 0.009$)$, and had longer time on mechanical ventilation (14.7 days vs 3.8 days, $p$ value $<0.001$ ) (Supplementary table S5). Secondly, we evaluated if the cause of death in patients with COVID-19/ influenza was directly or indirectly related or unrelated to the virus. Of all the deaths, $98 \%$ of deaths were directly or indirectly due to COVID-19 as and $89 \%$ of deaths were directly or indirectly due to influenza (supplementary table S6).

\section{DISCUSSION}

COVID-19 has been compared to influenza by both health care professionals and the lay public ${ }^{12-16}$ but limited detailed objective data are available for comparing and contrasting the impact of these two disease processes on patients and hospitals. We found that admissions for COVID-19 over a 2-month period at our medical center were more than double the total number of admissions for influenza during any 8-month influenza season in the past 5 years. In addition to the larger volume of cases within a much shorter time period, severity of illness and lethality for COVID-19 were also markedly higher than for influenza. We observed more mechanically ventilated COVID-19 patients (i.e., those with severe, life-threatening illness) in a 2-month period than occurred in five entire seasons of influenza combined, and a similar observation was made with patient deaths. Taken together, these findings indicate that COVID-19 causes more severe disease and is more lethal than influenza.

COVID-19 resulted in not only a marked increase in the number of mechanically ventilated patients in a short period of 
time but also a longer median duration of mechanical ventilation within this population. Specifically, 174 patients required mechanical ventilation for COVID-19 with a median duration on the ventilator of 14 days. In contrast, the combined seasons of influenza only resulted in 84 mechanically ventilated patients for a median duration of 3 days. This combination illustrates both the acute severity of disease and the prolonged nature of the respiratory failure that occurs in COVID-19 compared to influenza.

COVID-19 caused a substantial number of patients without major comorbid disease to require mechanical ventilation (44 people in 2 months). In contrast, influenza rarely led to mechanical ventilation in patients without underlying comorbidities (3 people over five seasons). In this specific subgroup, five patients with COVID-19 died in the 2-month period compared to one with influenza over 5 years. Pneumonia and acute respiratory distress syndrome were the predominant causes of mechanical ventilation for COVID-19 (94\%), whereas this was not the case for influenza $(56 \%)$. With influenza, the need for mechanical ventilation often developed as a result of exacerbation of a pre-existing health condition such as asthma or chronic obstructive pulmonary disease (COPD), which did not occur in COVID-19. The apparently higher rate of pneumonia and ARDS in our COVID-19 cohort may at least partly explain the more frequent occurrence of respiratory failure and death even in the absence of serious comorbidities.

In addition to acute respiratory failure, rates of vasopressor and renal replacement therapy were significantly increased in COVID-19 compared to influenza. The increased disease severity reflected in the rates of acute respiratory failure and other-organ injury paralleled the overall higher lethality of COVID-19 which resulted in 119 deaths in 2 months compared to 34 deaths from influenza over five seasons. The disparity in deaths was driven by both increased volume of cases and increased lethality within that volume in COVID-19 patients. This study did not evaluate organ injuries such as liver failure, neurological injury, and coagulation disorders, all of which have been reported with COVID-19. ${ }^{17-23}$

The increase in volume of COVID-19 cases compared to influenza is noted in Figure 2. This increase in patient volume occurred despite the implementation of increasingly stringent social distancing in Boston starting on March 15, 2020, ${ }^{24}$ and a statewide stay-at-home advisory starting on March 24, $2020 .{ }^{25}$ New cases began to decrease approximately 6 weeks after these measures were taken, though with resurgence of second wave number of cases in Boston started increasing in the month of November. Thus, the overall volume of cases of COVID-19 in this report was likely modified by these measures, whereas these mitigation measures were not taken during any influenza season. Influenza, however, can be modified by the implementation of widespread vaccination programs which are currently not available for COVID-19. Conversely, we are comparing a new pandemic due to COVID-19 to a well-studied endemic influenza entity.
While not a focus of this study, the critical care resources required to manage the marked increase and severity of disease in COVID19 included the emergent conversion of surgical and cardiac intensive care units to medical intensive care units, and the conversion of post-anesthesia care units and multiple medical wards to intensive care units. The conversion of any such space to an intensive care unit has not been done for any influenza season. In addition to space conversion, human resources (i.e., staffing models for new intensive care units), personal protective equipment, and increased use of dialysis were all part of the hospital-wide response to the COVID-19 pandemic but we did not compare these to influenza. Future studies may benefit from direct comparisons of resource utilization for the two disease processes.

Our study was limited by the evaluation of subjects in one hospital system, though this methodology allowed for granular assessments of differences between diseases. In addition, the true impact of COVID-19 in the absence of social distancing measures remains unclear as these measures were taken early and within weeks of the onset of hospital admissions. This study was conducted on a cohort of symptomatic COVID-19 patients admitted during the early period of the pandemic in the USA and during early surge of cases in Boston. Our admission criteria have not changed over time and we did not admit patients who were asymptomatic. However, some treatment practices have evolved over time such as the increased use of dexamethasone in COVID-19 patients.

\section{CONCLUSIONS}

COVID-19 resulted in significantly more weekly hospital admissions over 2 months than in five seasons of influenza in a region with community spread of both diseases. In addition, COVID-19 resulted in a significant increase in the use of mechanical ventilation, an increase in the use of vasopressor support, an increase in the incidence of renal injury, and an increase in mortality. COVID-19 was also significantly more likely to result in mechanical ventilation for those without major comorbidities as compared to influenza.

Acknowledgements: The authors would like to thank Lethu A. Ntshinga, AB, Jacob Boise, BS, Emma Hershey, BA, Mahmoud S. Issa, MD, Ying K. Loo, BS, and Natia Peradze, $M D, P h D$, for their data abstraction and data entry.

Corresponding Author: Michael W. Donnino, MD; Center for Resuscitation Science, Department of Emergency Medicine, Beth Israel Deaconess Medical Center, Boston, MA, USA (e-mail: mdonnino@bidmc.harvard.edu).

Funding The study was supported with internal funds. Dr. Donnino's effort is supported, in part, by grants from the National Institutes of Health (K24HL127101, R01HL136705, and 1RO1DK112886 and R03AA026093). Dr. Moskowitz is supported, in part, by a grant from the National Institutes of Health (K23GM128005). Dr. Berg is 
supported, in part, by a grant from the National Institutes of Health (5K23HL12881404).

\section{REFERENCES}

1. Faust JS, Del Rio C. Assessment of Deaths From COVID-19 and From Seasonal Influenza. JAMA Intern Med. 2020.

2. United States Department of Health and Human Services, Center for Disease Control. Past Seasons Estimated Influenza Disease Burden [Internet]. 2020 [cited June 12]; available from: https://www.cdc.gov/ flu/about/burden.htm. Accessed July 2020.

3. Kalil AC, Thomas PG. Influenza virus-related critical illness: pathophysiology and epidemiology. Crit Care 2019;23(1):258.

4. Ángeles-Sistac D, Martin-Onraet A, Cornejo-Juárez P, Volkow P, Pérez-Jimenez C, Vilar-Compte D. Influenza in patients with cancer after 2009 pandemic AH1N1: An 8-year follow-up study in Mexico. Influenza Other Respir Viruses 2020;14(2):196-203.

5. Guandelacy TM, Viboud C, Charu V, Lipsitch M, Goldstein E. Age- and sex-related risk factors for influenza-associated mortality in the United States between 1997-2007. Am J Epidemiol 2014;179(2):156-67.

6. Kunisaki KM, Janoff EN. Influenza in immunosuppressed populations: a review of infection frequency, morbidity, mortality, and vaccine responses, Lancet Infect Dis 2009;9(8):493-504.

7. Puig-Barberà J, Natividad-Sancho A, Trushakova S, Sominina A, Pisareva M, Ciblak MA, et al. Epidemiology of Hospital Admissions with Influenza during the 2013/2014 Northern Hemisphere Influenza Season: Results from the Global Influenza Hospital Surveillance Network. PLoS One 2016;11(5):e0154970.

8. Liu WD, Yeh CY, Shih MC, Sheng WH. Clinical manifestations and risk factors for mortality of patients with severe influenza during the 20162018 season. Int J Infect Dis. 2020;95:347-51. doi: https://doi.org/10. 1016/j.jjid.2020.04.013.

9. Kim Y-J, Lee ES, Lee Y-S. High mortality from viral pneumonia in patients with cancer. Infect Dis (Lond) 2019;51(7):502-9.

10. Kostova D, Reed C, Finelli L, Cheng PY, Gargiullo PM, Shay DK, et al. Influenza Illness and Hospitalizations Averted by Influenza Vaccination in the United States, 2005-2011. PLoS One. 2013;8(6):e66312. doi: https:// doi.org/10.1371/journal.pone.0066312.

11. United States Department of Health and Human Services, Center for Disease Control. Coronavirus Disease (2019) [Internet]. 2020 [cited June 12]; available from: https://www.cdc.gov/coronavirus/2019-ncov/ cases-updates/cases-in-us.html. Accessed July 2020.

12. Tolksdorf K, Buda S, Schuler E, Wieler LH, Haas W. Influenza-associated pneumonia as reference to assess seriousness of coronavirus disease (COVID-19). Euro Surveill 2020;25(11):2000258.

13. Tang X, Du RH, Wang R, Cao TZ, Guan LL, Yang CQ, et al. Comparison of Hospitalized Patients With ARDS Caused by COVID-19 and H1N1.
Chest. 2020;158(1):195-205. doi: https://doi.org/10.1016/j.chest.2020. 03.032 .

14. Rettner R. How does the new coronavirus compare with the flu? [Internet] 2020 [cited June 15]; available from: https://www.livescience. com/new-coronavirus-compare-with-flu.htm. Accessed July 2020.

15. Moore K, Lipsitch M, Barry JM, Osterholm MT. COVID-19: The CIDRAP Viewpoint. [Internet] 2020 [cited June 15]; available from: lhttps://www.cidrap.umn.edu/sites/default/files/public/downloads/ cidrap-covid19-viewp. Accessed July 2020.

16. Groth L. Is the Coronavirus Worse Than the Flu? Here's How the 2 Illnesses Compare [Internet] 2020 [cited June 15]; available from: https://www.health.com/condition/infectious-diseases/coronavirusworse-than-flu. Accessed July 2020.

17. Zhang C, Shi L, Wang F-S. Liver injury in COVID-19: management and challenges. Lancet Gastroenterol Hepatol 2020;5(5):428-30.

18. Del Rio C, Malani PN. COVID-19-New Insights on a Rapidly Changing Epidemic. JAMA. 2020;323(14):1339-40. doi:https://doi.org/10.1001/ jama.2020.3072.

19. Richardson S, Hirsch JS, Narasimhan M, Crawford JM, McGinn T, Davidson KW, et al. Presenting Characteristics, Comorbidities, and Outcomes Among 5700 Patients Hospitalized With COVID-19 in the New York City Area. JAMA. 2020.

20. Terpos E, Ntanasis-Stathopoulos I, Elalamy I, Kastritis E, Sergentanis TN, Politou M, et al. Hematological findings and complications of COVID-19. Am J Hematol 2020;95(7):834-47.

21. Guan WJ, Ni ZY, Hu Y, Liang WH, Ou CQ, He JX, et al.China Medica Treatment Expert Group for Covid-19. Clinical Characteristics of Coronavirus Disease 2019 in China. N Engl J Med. 2020;382(18):1708-20. doi: https://doi.org/10.1056/NEJMoa2002032.

22. Richardson S, Hirsch JS, Narasimhan M, Crawford JM, McGinn T, Davidson KW, et al. Presenting Characteristics, Comorbidities, and Outcomes Among 5700 Patients Hospitalized With COVID-19 in the New York City Area. JAMA. 2020;323(20):2052-59. doi: https://doi.org/ $10.1001 / \mathrm{jama} .2020 .6775$. Erratum in: JAMA. 2020 May 26;323(20):2098.

23. Helms J, Kremer S, Merdji H, Clere-Jehl R, Schenck M, Kummerlen C, et al. Neurologic Features in Severe SARS-CoV-2 Infection. N Engl J Med 2020;382(23):2268-70.

24. City of Boston. Coronavirus Disease (COVID-19) Timeline [Internet] 2020 [cited June 12]; available from: https://www.boston.gov/departments/ public-health-commission/coronavirus-timeline.

25. Mao L, Jin H, Wang $\mathbf{M}$, Hu $\mathbf{Y}$, Chen S, He $\mathbf{9}$, et al. Neurologic Manifestations of Hospitalized Patients With Coronavirus Disease 2019 in Wuhan, China. JAMA Neurol. 2020;77(6):683-90. doi: https://doi. org/10.1001/jamaneurol.2020.1127.

Publisher's Note: Springer Nature remains neutral with regard to jurisdictional claims in published maps and institutional affiliations. 